Research Paper

\title{
An Anti-Infection Tissue-Engineered Construct Deliv- ering Vancomycin: its Evaluation in a Goat Model of Femur Defect
}

Zhengqi Chang"\#,1,2,3,4, Tianyong Hou ${ }^{\#, 1,2,3}{ }^{\bowtie}$, Xuehui $\mathrm{Wu}^{1,2,3}$, Fei Luo ${ }^{1,2,3}$, Junchao Xing1,2,3, Zhiqiang Li1,2,3, Qianbo Chen ${ }^{1,2,3}$, Bo Yut $\mathrm{u}^{1,2,3}$, Jianzhong $\mathrm{Xu}^{1,2,3}$, Zhao $\mathrm{Xie}^{1,2,3} \bowtie$

1. National \& Regional United Engineering Lab of Tissue Engineering, Department of Orthopaedics, Southwest Hospital, the Third Military Medical University, Chongqing, China.

2. Center of Regenetive and Reconstructive Engineering Technology in Chongqing City, Chongqing, China.

3. Laboratory of Tissue Engineering in Chongqing City, Chongqing, China.

4. Department of Orthopedics, General Hospital of Jinan Military Commanding Region, Jinan, China.

\# These two authors equally contributed to this work.

$\square$ Corresponding authors: Tel: 86-023-68754164; fax: 86-023-65340297 Email: tianyonghou@126.com (Tianyong Hou), jianzhong_xu@126.com (Jianzhong Xu), xiezhao54981@yahoo.com.cn (Zhao Xie).

( ) Ivyspring International Publisher. This is an open-access article distributed under the terms of the Creative Commons License (http://creativecommons.org/ licenses/by-nc-nd/3.0/). Reproduction is permitted for personal, noncommercial use, provided that the article is in whole, unmodified, and properly cited.

Received: 2013.03.21; Accepted: 2013.10.06; Published: 2013.10.15

\begin{abstract}
A tissue-engineered construct (TEC) has previously been used for treating bone defects due to its strong osteogenic capability. However, transplantation of a TEC involves an open surgery that can cause infection. To overcome the potential risk of infection after TEC transplantation, we designed a system for the controlled release of antibiotics using fibrin gel-coated vancomycin alginate beads ( $F G-V a n c o-A B$ ) that can supply sustained antibiotics at the graft site. A TEC with FG-Vanco-AB was transplanted into critically sized bone defects of the right femur in a goat. As a control, the TEC without FG-Vanco-AB was transplanted into the left femur defect of the same goat. The breakpoint sensitivity of vancomycin for $S$. aureus $(5 \mathrm{mg} / \mathrm{L})$ was used as a known standard. Study results showed that the duration of time with vancomycin concentrations greater than $5 \mathrm{mg} / \mathrm{L}$ in the right graft site, blood, and left graft site were 28 days, 7 days, and 2 days, respectively. The bioactivity regarding vancomycin release was analysed by antibiotic disc diffusion. The vancomycin concentration was decreased from the centre of the graft to both ends of the femur. Radionuclide bone imaging showed no significant difference between the right and left TECs at either 28 or 56 days post-operation. Computed tomography and histological observation showed both sides' bone defects were healed by TEC at 112 days post-operation, and there was no significant difference in computed tomography value. These results suggest that FG-Vanco-AB in transplanted bone provided the ability to kill bacteria in local bone tissue while not interfering with the process of bone reconstruction and wound healing.
\end{abstract}

Key words: Tissue-engineered construct; controlled release; anti-infection; bone reconstruction.

\section{Introduction}

The tissue engineered construct (TEC) has achieved good results in repairing large bone defects due to its strong osteogenic capability [1-3]. However, treating bone defects or bone fusion involves open surgery, which have long air exposure times and po- tential for surgical injury that can easily lead to infection. However, if the blood supply at the injury site is sufficient, this issue can be effectively handled [4]. When using TEC to treat bone defects, the implanted TEC are devitalised tissues with no blood supply, 
making it an attractive habitat in which bacteria can live and breed [5]. Subsequently, both seeded cell death and serious infection could be induced around the graft site. These events explain why the infection rate after bone grafting is higher than after general orthopaedic surgery. Previous studies' documented infection rates after implanting an inactive allograft can be as high as $10 \sim 16 \%$, while rates in general orthopaedic surgery were $1 \%[4,6,7]$. Once infection occurs, it is often followed by very serious consequences that could lead to bone graft failure and poor patient outcomes, including increased morbidity, repeated operations, and long-term antibiotic treatment $[8,9]$. Thus, TEC use has been limited in clinical settings despite its enhanced osteogenic effect.

Some studies on in vivo implantation of TEC found that these sites undergo a gradual vascularisation process, developing from the edge to centre, before osteogenesis occurs [10]. Vascularisation is extremely important for the TEC because new blood vessels transport cells, factors, and nutrients to participate in bone repair and reconstruction [11,12]; new blood vessels also play a vital role in the anti-infection ability of tissues being supplied by them. Mononuclear blood cells can engulf and decompose foreign microorganisms, and plasma components such as anti-toxin, lysozyme and other factors that are able to resist and eliminate invasive bacteria and toxins. In addition, new blood vessels are essential for transporting antibiotics and other drugs to infection sites to elicit bactericidal effects [13].

Through the above analysis, we find that implanted TEC have a high risk of infection before vascularisation and that providing the TEC with antibacterial ability before vascularisation is important to avoid infection. Hou et al. [14] demonstrated that TEC with fibrin gel-coated vancomycin alginate beads (FG-Vanco-AB) did not affect the proliferation and differentiation of seeded cells in vitro. Moreover, we found that the reported release time of FG-Vanco-AB was consistent with the time for TEC vascularisation in vivo [14-16]. Provided that an anti-infection TEC can be constructed and implanted to maintain good osteogenic capacity with no or minimal influence on osteogenesis, the potential infection of the TEC could be controlled prior to completing vascularisation.

Chan et al. [17] confirmed the efficacy of a local, controlled release of antibiotics in preventing bone infection [18]. Furthermore, vancomycin-alginate controlled release systems $[14,19]$ have been reported with optimal release times, biosecurity and bacterial properties in vitro. Based on these studies and to address the issue of infection in the TEC, we utilised the alginate-vancomycin controlled release system by setting up FG-Vanco-AB [20]. To test this system, twenty millimetre osteoperiosteal segmental defects (precisely sized bone defects) were made in the mid-diaphyseal region of both femurs from test animals, and these segments were stabilised by static intermedullary rods (sIMR). FG-Vanco-AB and tissue engineered construct containing autogenic bone marrow-derived mesenchymal stem cells (BMSCs) and allogeneic demineralised bone matrix (DBM) was used to fill the defect in the right femur. As a control, the TEC without FG-Vanco-AB was used to fill the defect in the left femur of the same subject. The aggregate analysis of local vancomycin release, TEC blood supply, and bone defect repair was detected by high-performance liquid chromatography (HPLC), radionuclide bone imaging, computed tomography (CT), and histological and tracing observations.

\section{Materials and Methods}

\section{I Preparation of vancomycin alginate beads}

Vancomycin alginate beads (Vanco-AB) were prepared following a previously published method [14]. In brief, a mixture composed of equal volumes of sterile vancomycin solution $(50 \mathrm{mg} / \mathrm{mL}$, Eli Lilly, Indianapolis, Indiana) and $16 \%(\mathrm{w} / \mathrm{v})$ sterile sodium alginate solution (low viscosity, Sigma, St. Louis, MO) were added dropwise into a $1 \mathrm{mM} \mathrm{CaCl}_{2}$ (SinoReagent, Shanghai, China) solution with gentle stirring. After the beads were allowed to set for $10 \mathrm{~min}$, they were washed with sterile deionised water and lyophilised for at least $8 \mathrm{~h}$. The beads were alternatingly immersed in a $75 \mathrm{mg} / \mathrm{mL}$ fibrinogen (Guangzhou Bioseal Biotech CO., LTD, China) solution for ten minutes and a $400 \mathrm{U} / \mathrm{mL}$ thrombin (Guangzhou Bioseal Biotech CO., LTD, China) solution for ten seconds. The resulting fibrin gel-coated vancomycin alginate beads, abbreviated as FG-Vanco-AB [20], were kept at $-70^{\circ} \mathrm{C}$ for $6 \mathrm{~h}$, freeze-dried at low pressure for $12 \mathrm{~h}$ until reaching a constant weight, and stored at $4^{\circ} \mathrm{C}$ until use.

\subsection{Cell isolation, expansion, and labelling by enhanced green fluorescent protein}

BMSCs were isolated from the iliac marrow of adult male goats according to a previous study [14], which was approved by the Animal Care and Use Committee of the Third Military Medical University (Chongqing, China). Nucleated cells were isolated by a density gradient solution (Histopaque ${ }^{\circledR}$, Sigma, St. Louis, MO) and plated at a density of $10^{5}$ cells $/ \mathrm{mL}$ in a culture flask in standard medium: Dulbecco's modified Eagle's medium/Hamm's Nutrient Mixture F12 (1:1) (DMEM/F12, Hyclone) with 10\% foetal bovine serum (FBS, Hyclone), $100 \mathrm{U} / \mathrm{mL}$ penicillin (Sigma, St. Louis, MO) and $100 \mu \mathrm{g} / \mathrm{mL}$ streptomycin (Sigma). 
Passage 3 cells were used in this study.

BMSCs were transfected with the retrovirus vector pLEGFP-N1 (Clontech, Mountain View, CA) with enhanced green fluorescent protein (EGFP), as described by Hou et al. [3]. BMSCs expressing EGFP were picked and seeded into a new culture flask for further culture.

\subsection{Fabrication of tissue-engineered construct}

DBM was manufactured by previously published methods with modifications [3]. Goat cancellous bone was harvested from the femoral condyles of 2- to 3-year-old goats according to the regulations of the Animal Care and Use Committee of the Third Military Medical University (Chongqing, China). After residual lipid tissue was removed, the samples were sectioned into cubes $(10 \mathrm{~mm} \times 10 \mathrm{~mm} \times 5 \mathrm{~mm})$ and demineralised. DBM scaffolds were processed by vacuum freeze-drying, vacuum packaging and irradiation sterilisation. These samples were typically stored at $-70^{\circ} \mathrm{C}$ for at least 3 months before use.

The DBM scaffolds were submerged in DMEM/F12 medium overnight at $4^{\circ} \mathrm{C}$ before the fabrication of the TEC, and then, they were dislodged from the medium and air dried. The BMSCs were redissolved in DMEM/F12 medium with 20\% FBS at a cell density of $10^{7}$ per millilitre. A $0.1 \mathrm{~mL}$ cell suspension was seeded to each side of DBM on two 10 $\mathrm{mm} \times 10 \mathrm{~mm}$ sides. After a 2-hour incubation, the other side of the DBM was prepared in the same way. After another 2 hours, the prepared TEC was immersed in osteogenic medium for further culture. The osteogenic medium was composed of standard medium plus 100 $\mathrm{nM}$ dexamethasone (Sigma, St. Louis, MO), $50 \mu \mathrm{M}$ ascorbic acid 2-phosphate (Sigma) and $10 \mathrm{mM}$ $\beta$-glycerophosphate (Sigma, St. Louis, MO). The TEC was cultured for 7 days prior to surgical implantation.

\subsection{Surgical procedure and postoperative management}

The goat animal models were anaesthetised with $0.06 \mathrm{ml} / \mathrm{kg}$ Sumianxin II (Junxu University of PLA, Changchun) and $2 \mathrm{mg} / \mathrm{kg}$ ketamine hydrochloride (Hengrui Medicine Ltd. Company, Jiangsu). Twenty millimetre osteoperiosteal segmental defects (critically sized bone defects) were made in the mid-diaphyseal region of both femurs according to a previously published method [3]. Stabilisation was sustained by static intermedullary rods (sIMR). Tissue-engineered construct containing autogenic BMSCs and allogeneic DBM was washed with DMEM medium to remove bovine serum prior to implantation. A composite of the TEC and $1 \mathrm{~g}$ FG-Vanco-AB was used to fill the defect in the right femur (experimental group, EXP group). As a control, the TEC without FG-Vanco-AB was used to fill the defect in the left femur (control group, CON group). Eighteen goats were conditioned as models for antibiotics analysis and histological observation.

\subsection{Analysis of the concentration and bioac- tivity of vancomycin}

Tissue fluid from the graft area of both femurs was collected by syringe at three different sites to avoid nonuniform vancomycin injection concentrations at 1, 2, 3, and $7 \mathrm{~d}$ post-operation. Six goats were euthanised according to regulations of the Animal Care and Use Committee of the Third Military Medical University (Chongqing, China) on 14, 28, and 112 d post-operation. Both femurs were sliced into $5 \mathrm{~mm}$ sections at $10 \mathrm{~mm}$ intervals beginning at the graft; seven slicing sites were selected for detecting vancomycin concentration and were used to evaluate the diffusion of vancomycin in the bone tissue. Another two sites were located at the two ends of the femur. In brief, the slice was measured for volume and then powdered. Additionally, $5 \mathrm{~mL}$ of a $0.9 \%$ sodium chloride solution was dropped to resolve vancomycin in the slice by gentle stirring. After centrifugation for $10 \mathrm{~min}$ at 10,000 rpm, the supernatant was collected and freeze-dried to remove liquid. The same volume as the original tissue of either $0.9 \%$ sodium chloride solution or goat serum was used for resolving vancomycin. The goat serum was collected concurrently.

The concentrations of vancomycin in the solution and serum were determined by measuring the peak area by HPLC analysis with a Waters 510 Multisolvent Delivery System and Discovery C18 column (4.6 mm $\times 250 \mathrm{~mm}$, Supelco, Sigma-Aldrich Corporation, USA) according to methods detailed by Hou et al. [14]. The mobile phase was acetonitrile (Tedia Company Inc., USA) and $40 \mathrm{mM} \mathrm{NaH} \mathrm{PO}_{4}(11 / 89$, $\mathrm{v} / \mathrm{v})$, and the flow rate was $1.0 \mathrm{~mL} / \mathrm{min}$. The wavelength for detection was set at $236 \mathrm{~nm}$.

The bioactivity of vancomycin from different tissues (tissue fluid, bone, and serum) was analysed by antibiotic disc diffusion, as described by Ueng et al. [19]. Eight microlitres of the sample from redissolved vancomycin solution and goat serum was pipetted onto the 7-mm discs. The discs were placed on nutrient agar plates (beef extract, peptone, agar) that were then seeded with a lawn of Staphylococcus aureus (ATCC 25923), and zones of inhibition were measured via micrometry after 18 hours of incubation at $35^{\circ} \mathrm{C}$.

\subsection{Serum creatinine and urea nitrogen}

The data for urea nitrogen (UREA, mmol/L) and creatinine (CRE, $\mu \mathrm{mol} / \mathrm{L}$ ) from four operative goats were consistently collected and analysed on days $1,2,3,7,14,21$, and 28 postoperation. Their 
values were analysed and compared with pre-operation values for evaluating potential kidney damage.

\subsection{Radionuclide bone imaging}

Six goats underwent analysis of radionuclide bone imaging at 4 and 8 weeks after operation according to previously published methods [3]. In brief, all goats were analysed by three-phase bone scintigraphy 3 hours after injection of $2 \mathrm{mCi} / \mathrm{kg}$ technetium-99m methylene diphosphonate (99mTc-MDP, Beijing Atom-Hitech Company, China). Images were collected from the posterior surface of the hind limbs by a Millennium/MPR single-photon emission computerised tomography (SPECT) (GE, Piscataway, NJ, USA) and were analysed with the eNTEGRA workstation. Blood pool images were obtained at a rate of one picture per second; bone scan images were obtained at a rate of one picture per five minutes. Blood pool and bone scan images were 15 to 20 minutes after starting points of 5 minutes and 4 hours after radioisotope administration, respectively. All studies were evaluated visually and semi-quantitatively. A square region of interest (ROI) was drawn for the EXP (with FG-Vanco-AB) and CON (without FG-Vanco-AB) groups, and the mean value of 3 pictures from both femurs was used for comparison.

\subsection{Computed tomography scan, Histological observation and BMSCs tracing}

After euthanasia at 14, 28, and $112 \mathrm{~d}$ postoperation, femurs from goats were excised for images using a spiral computed tomography (CT) multi-detector (Siemens, Germany). The 3-D images of femurs were reconstructed using a middle-frequency kernel at a 1-mm-thick axial scanning density. The CT value (Hounsfield unit) of the graft was defined as the mean of two ROIs in a 2-D middle longitudinal segment. A rectangular region of interest (ROI) was determined.

The grafts were sectioned into small bone blocks for histology and histomorphometry according to the procedures described below. Samples were fixed in $10 \%$ neutral buffered formalin for 48 hours, subjected to decalcification, dehydrated in graded alcohols, and embedded in paraffin. Then, samples were sectioned to a thickness of $5 \mu \mathrm{m}$ and stained with haematoxylin and eosin. Photomicrographs of sections were taken with an Olympus BX-60 light microscope. The decalcified samples were embedded in Tissue-Tek O.C.T., and $10-\mu \mathrm{m}$-thick sections were used for examination of EFGP-labelled MSCs in samples by a Leica TCS SP5 confocal laser scanning microscope (excitation at 488 $\mathrm{nm}$ ), and images were subsequently reconstructed by computer.

\subsection{Statistical analysis}

All data were presented as mean $\pm \mathrm{SD}$, and subjected to a paired Student's t-test using SPSS version 10.0 software package (SPSS Inc., Chicago, IL USA). Differences were considered significant at $P<0.05$.

\section{Results}

\section{I Fibrin gel-coated vancomycin alginate beads and tissue-engineered construct}

The Vanco-AB beads were spherical and white with a diameter of approximately $5 \mathrm{~mm}$. However, the size was reduced to approximately $3 \mathrm{~mm}$ after lyophilisation. Coating the fibrin gel made the Vanco-AB beads smoother, and their weight increased by approximately $8 \%$ (Fig. 1A). The beads were laid in the pores of TEC (DBM scaffold) before transplantation by centrifugation at $1000 \mathrm{rpm}$ for $3 \mathrm{~min}$ (Fig. 1B).

BMSCs were labelled with EGFP through transfection by a retroviral vector and then seeded into DBM scaffolds. At day 7, observation by scanning electron microscope (AMRAY-1000B, AMRAY) (Fig. 1C) and confocal laser scanning microscope (Leica TCS SP5, Wetzlar, Germany) (Fig. 1D-F) showed that BMSCs were distributed throughout the whole DBM scaffold.

\subsection{Structure of graft in vivo}

CT scan showed that there was no new bone formation in the graft zones of either femur at 14 days post-operation (Fig. 2A, 2D). At 28 days, new bone formation was observed in the graft zones of both EXP and CON groups, but this growth was interrupted, and there was no continuous new bone between the broken ends of the bone defect (Fig. 2B, 2E). At 112 days post-operation, bone defects in both groups were filled with new bone, and the broken ends of the femur were connected by continuous new bone (Fig. $2 \mathrm{C}, 2 \mathrm{~F})$. The CT value in both femurs' graft zones suggested that there was no significant deviation between the EXP and CON groups in each phase (Fig. $2 \mathrm{G})$.

H.E. staining at 14 days post-operation showed that there were some alginate materials in EXP group (Fig. 3A), and that they were distributed in DBM scaffolds or packaged in fibrous and muscle tissues. Tissue structure in the EXP group was similar to the $\mathrm{CON}$ group. However, there was no alginate in the CON group (Fig. 3D). Infiltration of granulation tissue and formation of capillary vessels were observed in both groups, accompanied by sporadic lymphocytes and polykaryocytes. At 28 days post-operation, a considerable number of osteoblasts-like cells were observed in DBM scaffolds in both groups, and there was a great deal of extracellular matrix in adjacent 
zones (Fig. 3B and E). A degradation product of the alginate materials was still observed in the EXP group (Fig. 3B). At 112 days post-operation, osteoblast-like cells were embedded in the extracellular matrix, forming bone tissue that resembled normal bone tissue structure in both groups (Fig. 3C and F). Alginate materials were not observed in the EXP group (Fig. $3 C)$.

The survival and distribution of implanted MSCs were observed in both EXP (Fig 4.A and D) and CON groups (Fig 4B and E). At 14 days post-operation, most implanted MSCs remained around the DBM scaffold and a few migrated into the adjacent host tissue (Fig 4.A and B). At 28 days (Fig 4.D and E) post-operation, the implanted MSCs distributed almost evenly along the newly formed bone, but few MSCs were observed in the host tissue.

\subsection{Assaying vancomycin in tissue fluid, blood and bone tissue}

Previous studies $[14,20]$ showed that the minimum inhibitory concentration, minimum bactericidal concentration, and breakpoint sensitivity of vancomycin for S. aureus (ATCC 25923) were $1.2 \mathrm{mg} / \mathrm{L}, 2.1$ $\mathrm{mg} / \mathrm{L}$ and $5 \mathrm{mg} / \mathrm{L}$, respectively. Based on the breakpoint sensitivity of vancomycin for S. aureus, the du- ration of time when the vancomycin concentration in the right femur grafted site was higher than $5 \mathrm{mg} / \mathrm{L}$ equalled 28 days. In the CON group, the vancomycin concentration was over $5 \mathrm{mg} / \mathrm{L}$ only on the second day post-operation. In the serum, the same situation (over $5 \mathrm{mg} / \mathrm{L}$ ) appeared during the 7th day post-operation but not on the 1st day (Fig 5A).

On the 14th day post-operation, sections from the EXP group showed that the highest vancomycin concentration appeared in the grafted tissue in the middle of femur, and the vancomycin concentration in the tissue gradually decreased from the middle to both ends of the femur. However, the vancomycin concentration in every site of the right femur (EXP group) was higher than the serum and left femur (CON group) concentrations at the same time point (Fig 5C.). Furthermore, vancomycin concentration in the serum and left femur (CON group) was not detectable on the 14th day post-operation.

The analysis of antibiotic disc diffusion for three samples from different tissues showed that the vancomycin concentration was consistent with the diameter of the inhibition zone, so higher vancomycin concentrations correlated with larger diameters of the inhibition zone (Fig 5B).
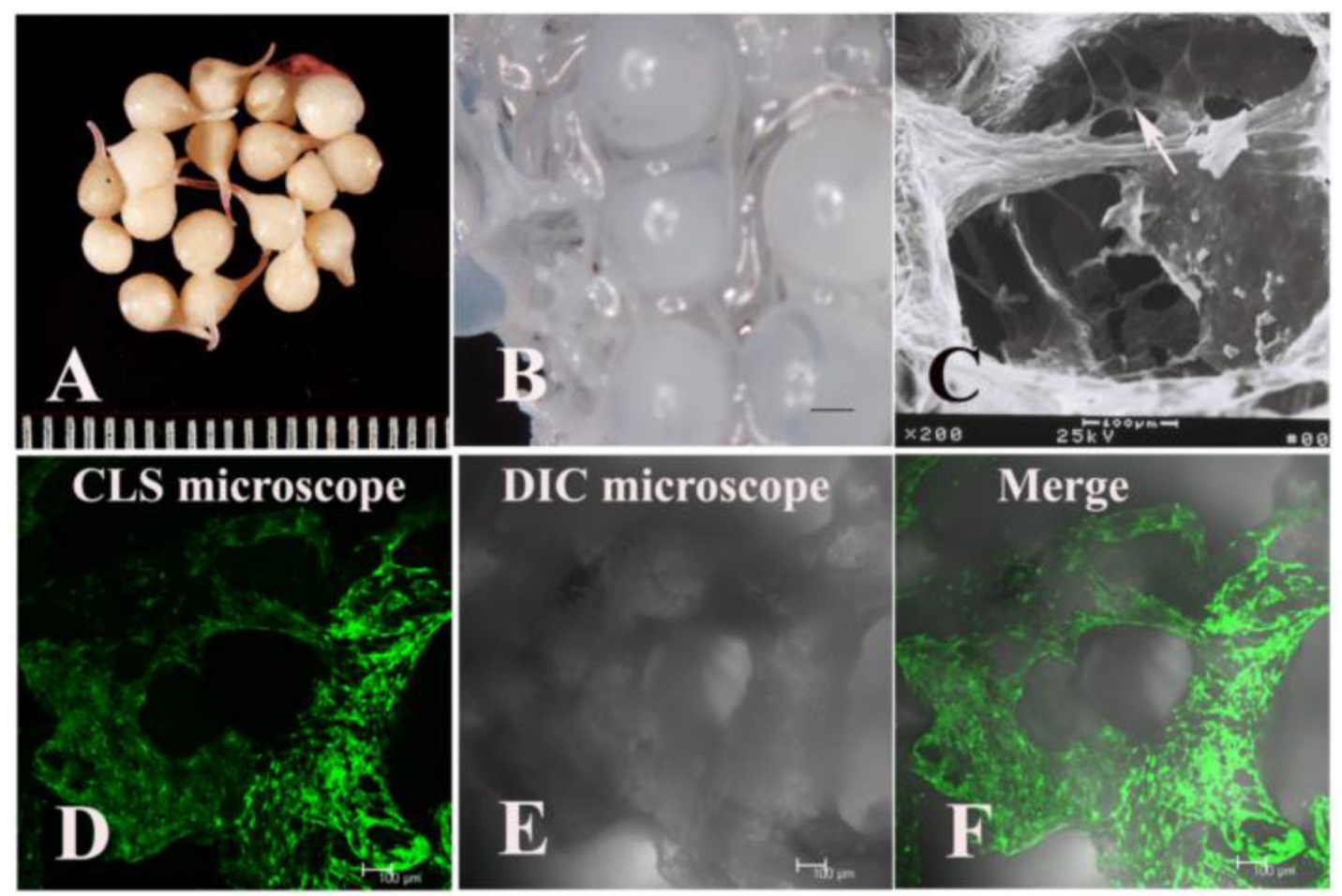

Fig I. The appearance of fibrin gel-coated vancomycin alginate beads (FG-Vanco-AB) (A). BMSCs were labelled with EGFP through transfection by a retroviral vector and then seeded into DBM scaffolds (B). At day 7, BMSCs (white arrow) were distributed throughout the whole DBM scaffold as observed by scanning electron microscopy $(C)$ and confocal laser scanning microscopy (D-F). 

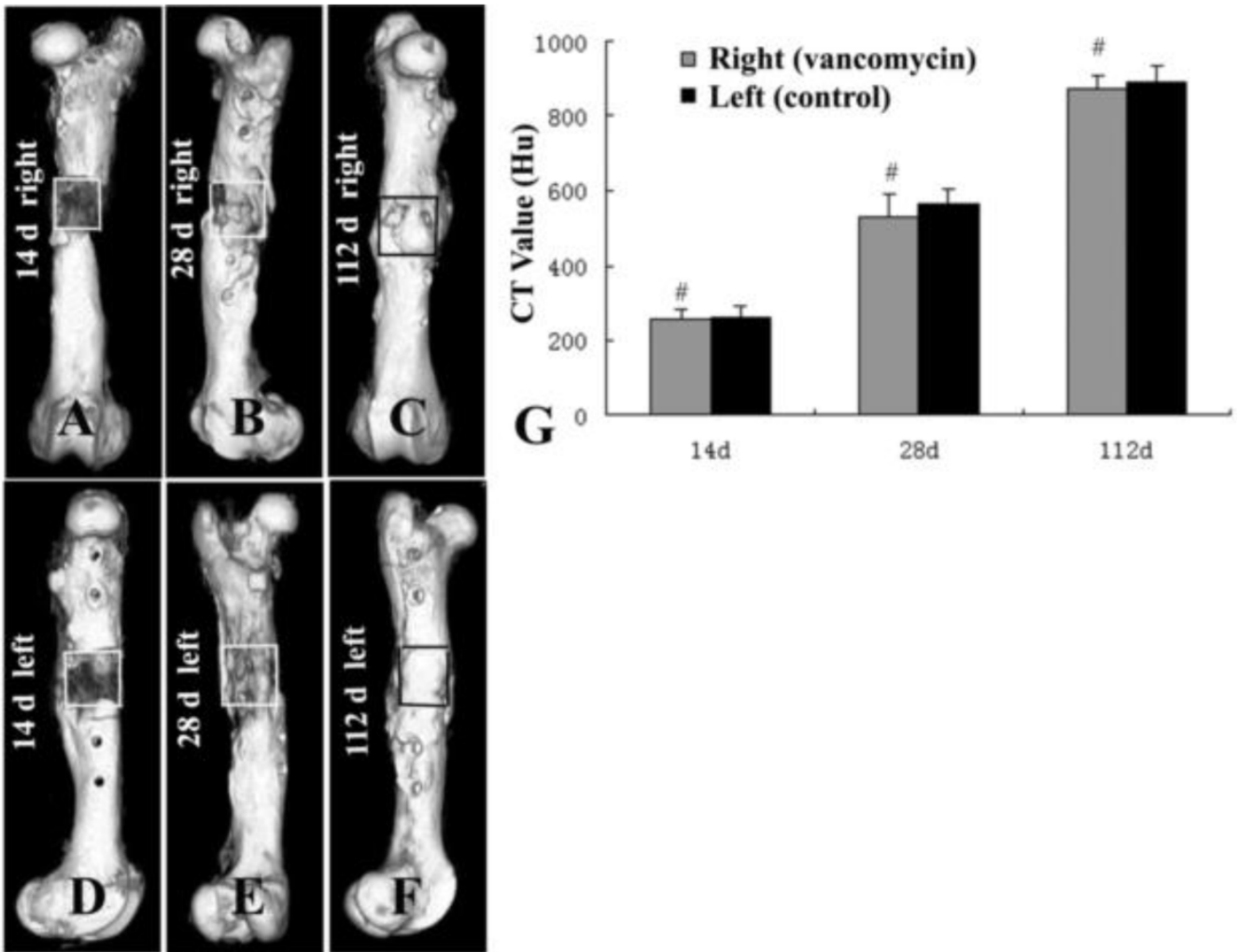

Fig 2. Computed tomography (CT) scan of the grafts in both groups at I $4(A, D), 28(B, E)$, and I I $2 \mathrm{~d}(\mathrm{C}, \mathrm{F})$ post-operation. CT values (G) in both groups suggested that there was no significant difference between the EXP and CON groups in each phase (\#, $P>0.05)$.

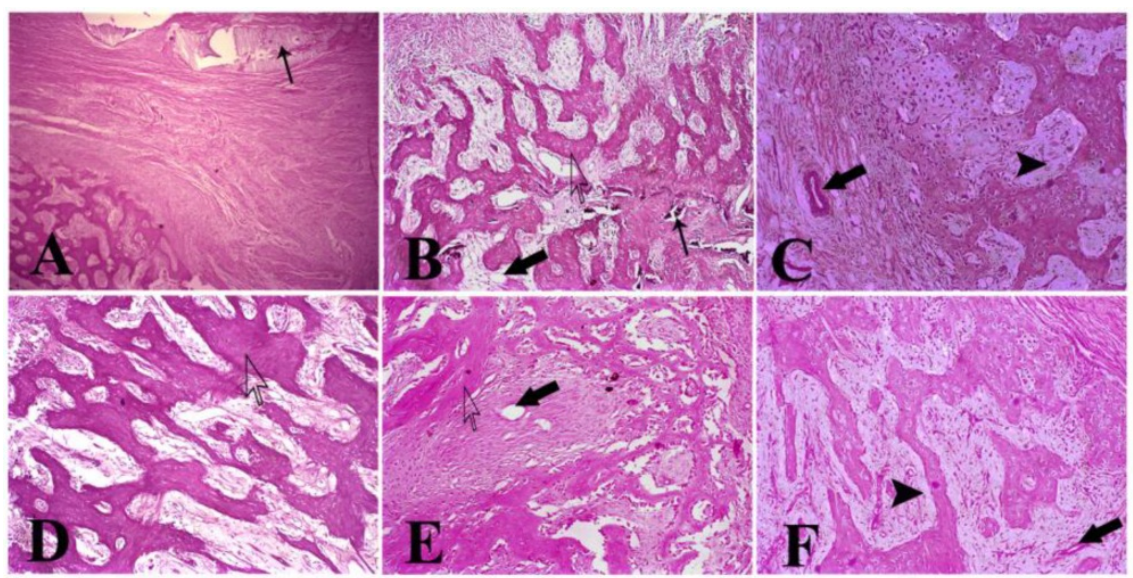

Fig 3. H.E. staining showed that there were some alginate materials (slim arrow) at I 4 days post-operation in the EXP group (A). There was no alginate material in the CON group (D). DBM scaffolds (hollow arrowhead) were observed in both groups. At 28 days post-operation, degradation product of alginate materials was still observed in the EXP group (B). Significantly increased numbers of osteoblast-like cells were observed in DBM scaffolds in both groups and in their adjacent zone a large amount of extracellular matrix (B and E). Blood vessels were also observed (gross arrow). At II2 days post-operation, osteoblast-like cells were embedded in the extracellular matrix and newly formed bone tissue (solid arrowhead) (C and F). The blood vessel was close to newly formed bone tissue. Alginate material was not observed in the EXP group $(A, \times 40 ; B-F, \times 100)$. 

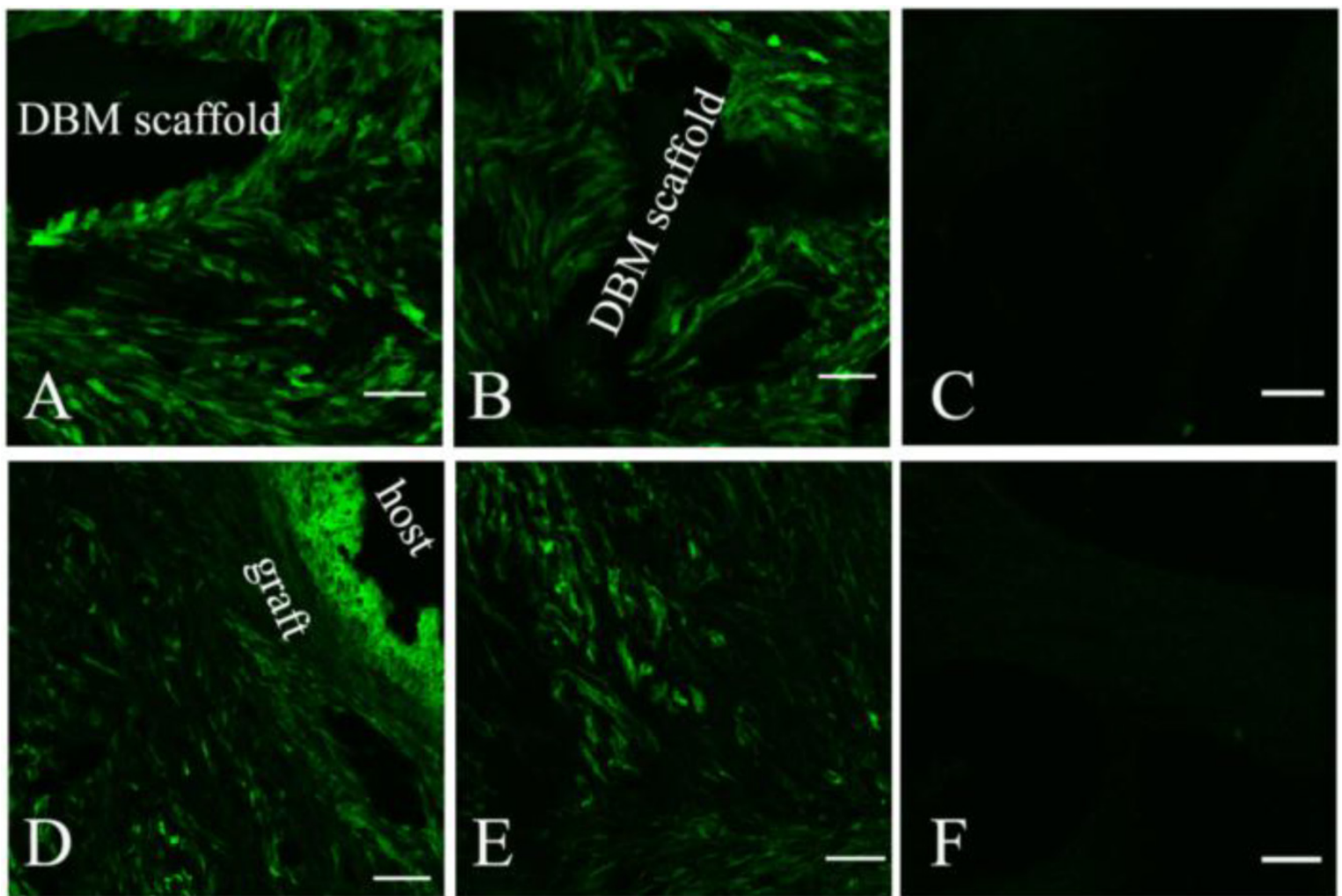

Fig 4. The survival and distribution of implanted MSCs were observed by confocal laser scanning microscope after tissue-engineered grafts fabricated by labelled MSCs and DBM were transplanted into the EXP (A and D) and CON (B and E) groups. An image scanned from normal bone tissue was used as a control ( $C$ and F). At 2 weeks post-operation, most implanted MSCs stayed around the DBM scaffold and a few migrated into the proximal tissue of the host (A and B). At 4 weeks ( $D$ and $E$ ) post-operation, the implanted MSCs distributed almost evenly along the newly formed bone, but few were observed in the host. Scale bar: A-C, $50 \mu \mathrm{m}$; D-F, $100 \mu \mathrm{m}$.

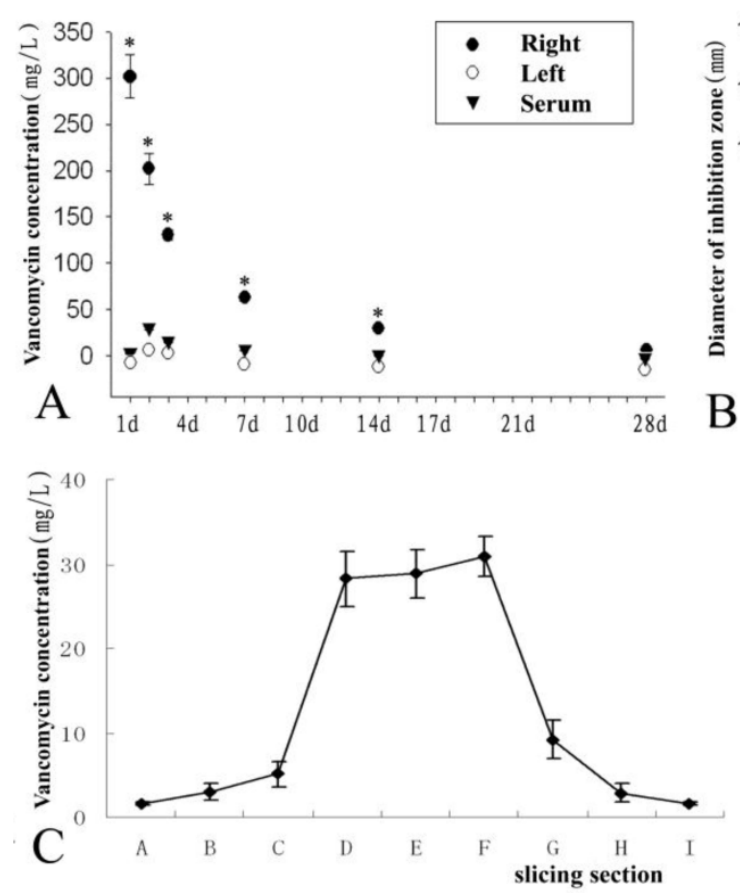

Fig 5. The vancomycin concentration was detected from different samples of the right graft (EXP group), the left graft (CON group), and serum (A). The diameter of the inhibition zone of the tissue fluid from the EXP group, CON group, and serum was analysed in (B). There was a trend of decreasing vancomycin concentration among EXP group, CON group, and serum in Figures A and B. On the 14th day post-operation, sections of the EXP group (see Figure I) showed the highest vancomycin concentration in the grafted tissue in the middle of the femur, while vancomycin concentration in the tissue gradually decreased from the middle to both ends of the femur $(C) . *$ represents a significant difference in each phase between the EXP and CON groups $(P<0.05)$ 


\subsection{Analysis of radionuclide bone imaging by SPECT}

Blood pool and bone scan images represented the blood supply of the bone tissue and bone viability, respectively. Among all blood pool and bone scan images, there were no significant differences in the blood pool and bone scan counts between the EXP and CON groups at 4 and 8 weeks post-operation (Fig. 6), suggesting that there were no significant differences in the blood supply of bone tissue or bone viability between the anti-infection TEC and the con- trol TEC $(P>0.05)$. However, the count at week 4 was significantly higher compared to week $8(P<0.05)$.

\subsection{Change in serum creatinine and urea ni- trogen}

When compared with the pre-operation levels, there were periodic changes in the serum creatinine (CRE) and urea nitrogen (UREA); however, the highest post-operative value did not exceed the highest pre-operative value (Fig. 7).

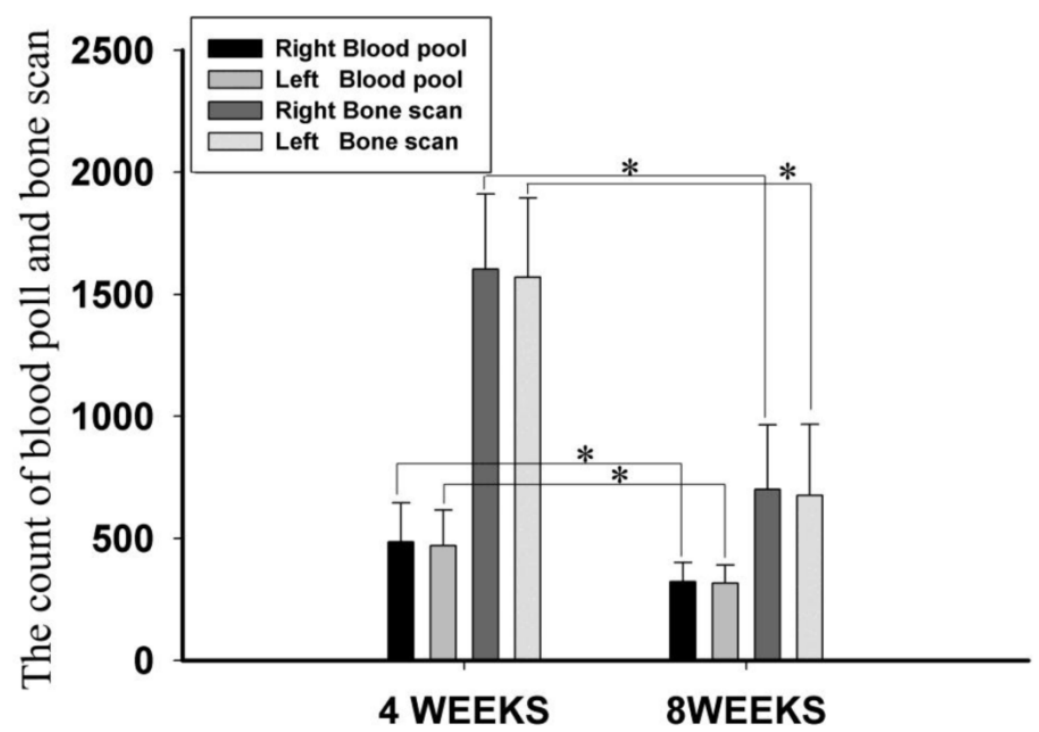

Fig 6. Blood pool and bone scan images. There was no significant difference in the count of blood pool and bone scan between EXP and CON groups at 4 or 8 weeks post-operation $(P>0.05)$. However, the count at week 4 was higher than that at week 8 , and this difference was significant $(P<0.05)$. ${ }^{*}$ represents a significant result $(P<0.05)$.

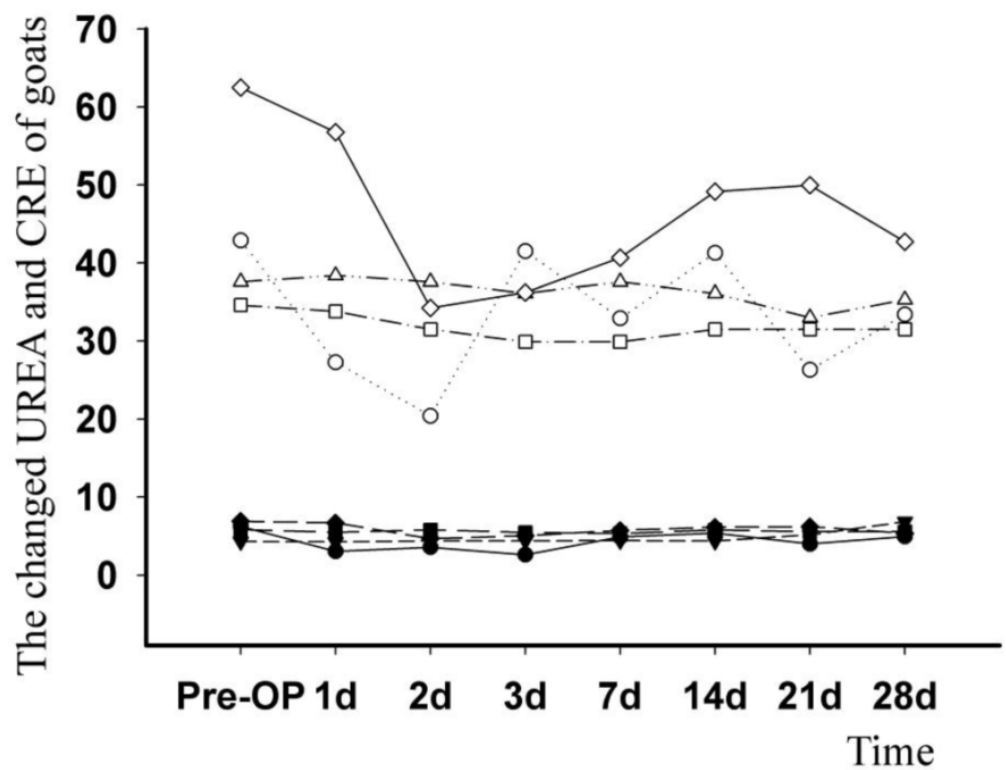

Fig 7. Data on urea nitrogen (UREA, stuffed symbol, mmol/L) and creatinine (CRE, aircored symbol, $\mu \mathrm{mol} / \mathrm{L}$ ) levels from four operative goats was persistently collected and analysed. The changed curve showed that there was a significant increase in both analytes when comparing post-operation to pre-operation levels. 


\section{Discussion}

TEC have been widely applied to large bone defects due to their excellent osteogenic effect, but multiple problems remain. During the surgical operation, the open incision is exposed to air for a long time and could result in potential infection by invasive bacteria [4]. Furthermore, implanted TEC have an insufficient blood supply, making the immune responses of the host ineffective at the transplantation site as well as obstructing the local delivery of antibiotics from the peripheral blood [5]. Therefore, TECs are the first and ideal site for pathogenic bacteria and opportunistic pathogens to invade, proliferate, and colonise. The success of a TEC implantation could be improed by preventing infection.

Sufficient blood supply is essential for the immune responses and antibiotic transmission from the host body as well as the maintenance of metabolism [13]. Thus, vascularisation of an implantation is crucial for inhibiting and killing bacteria, as well as for transporting cytokines and nutrients to participate in bone repair and reconstruction. However, vascularisation begins from the edges and gradually spreads centrally [10]. The vascularisation of the implanted TEC was essentially complete at 4 weeks post-operation, peaking at approximately 6 weeks. Subsequently, the vessels turn to the remodelling phase, during which the number of blood vessels begins to decline until normal $[15,16,21-23]$. There was consequently a high risk infection time window for implanted TEC up to 4 weeks post-operation. The incidence of infection at the TEC may be significantly reduced by equipping the TEC with stable bacteriostatic or bactericidal ability before the completion of basic vascularisation.

A previous study determined that alginate microspheres embedded with vancomycin could maintain an effective bactericidal concentration for approximately 4 weeks in vitro [14], which was consistent with the time required for basic vascularisation. Furthermore, alginate microspheres were also applied with an injectable tissue-engineered construct for treating bone infection [24]. These results showed that alginate microspheres embedded with vancomycin can be promising for preventing infection of the TEC before basic vascularisation, and even in healing an existing bone infection. Although the vancomycin content in the implants gradually decreased from 1 to 16 weeks post-operation, an effective bactericidal concentration was maintained up to 4 weeks post-operation. Consistent with this finding, addition of the implants' powder into petri dishes containing $S$. aureus resulted in antibacterial circles in all dishes with areas decreasing over implant time up to 4 weeks. HE staining also showed that the volume of microspheres reduced over time, while vascularisation and osteogenesis increased. Radionuclide bone imaging showed a vascularisation process similar to previous reports, with vascularisation of implanted TEC essentially completed by 4 weeks post-operation, and the volume of blood flow began to decrease at 8 weeks post-operation. Therefore, antibacterial TECs can reasonably be believed to have elicited anti-infection effects while encountering latent infringing bacteria before the establishment of vascularisation.

With vascularisation of the TEC, the release of antibiotics from microspheres was gradually reduced. The convergence of decreasing vancomycin release and increasing vascularisation was seamless, thus adequately inhibiting infection of the implants. Notably, the elimination of potential pathogens located in the surrounding host tissues of the TEC primarily depends on the sufficiency of the local bloodstream, not the controlled release microspheres. This case is similar to some ordinary orthopaedic surgeries [4] where the incidence of infection is very low. Antibiotics released from microspheres are quickly absorbed into the bloodstream, and the diffusion distance is confined. Hence, an anti-infection TEC may make a limited contribution to the anti-infection abilities of the surrounding tissue; however, our focus remains on the anti-infection ability of the TEC.

In groups of anti-infection TEC and normal TEC, osteogenesis was similar at 14, 28, and 112 days post-operation. Reconstruction of bone in both groups was accomplished by 112 days, as shown by CT scan. These results indicated that, consistent with in vitro data, the incorporation of alginate microspheres embedded with vancomycin had no additional impact on the osteogenic effects of TEC in vivo [14]. The upper limit of safe concentration of vancomycin was reported as $1,000 \mathrm{mg} / \mathrm{L}$ [25]; above this level, vancomycin would affect the proliferation and differentiation of cells, as well as induce cell death. For our TEC, the highest vancomycin concentration in the right graft was $329 \mathrm{mg} / \mathrm{L}$, a relatively secure range. Therefore, we concluded that the dose of vancomycin in all alginate microspheres was within a safe range and that there was no harmful effect on the seeded cells. Kidney damage is also a major side effect of vancomycin [26]. However, the vancomycin concentration in blood was far lower than the concentration in the graft. The continual analysis of serum creatinine and urea nitrogen showed that anti-infection TEC did not affect kidney function.

In contrast to previous studies [14, 24], this article mainly focused on the effect of FG-Vanco-AB on TEC osteogenesis and the effect of vancomycin release 
on the host. Evaluating the osteogenic ability and safety of the anti-infection TEC were the novel goals of the paper. First, FG-Vanco-AB was composed of absorbable biomaterials. They occupied some space within the implant before they were completely degraded, and this could affect osteogenesis of the TEC. Second, the safety of vancomycin in local application was unknown. Based on the conclusive anti-infection action of FG-Vanco-AB in a previous study [24], the osteogenic ability and safety of anti-infection TEC should be determined before planning its clinical application. Similar to TEC, anti-infection TEC with FG-Vanco-AB performs well with respect to osteogenic efficacy. Furthermore, anti-infection TEC can continuously release vancomycin, effectively preventing latent infection during the process of osteogenesis, and exhibits satisfactory biosecurity. In summary, the application of an anti-infection TEC may reduce the incidence of postoperative infection, and may greatly improve the success rate of bone grafts.

\section{Acknowledgements}

This work was supported by The National Natural Science Foundation of China (30900312, 81371975, 81071465), The Foundation of Young Innovative Talents of Third Military Medical University (2009XQN23), The National High Technology Research and Development (863) Program of China (2006AA02A122), and the Military Foundation (BWS11C040).

\section{Competing Interests}

The authors have declared that no competing interest exists.

\section{References}

1. Xie H, Yang F, Deng L, et al. The performance of a bone-derived scaffold material in the repair of critical bone defects in a rhesus monkey model. Biomaterials. 2007; 28: 3314-3324.

2. Viateau V, Guillemin G, Bousson V, et al. Long-bone critical-size defects treated with tissue-engineered grafts: a study on sheep. J Orthop Res. 2007; 25: 741-749.

3. Hou T, Li Q, Luo F, et al. Controlled dynamization to enhance reconstruction capacity of tissue-engineered bone in healing critically sized bone defects: an in vivo study in goats. Tissue Eng Part A.2010; 16: 201-212.

4. Ahn DK, Park HS, Choi DJ, et al. The difference of surgical site infection according to the methods of lumbar fusion surgery. J Spinal Disord Tech. 2012; 25: E230-234.

5. Day RE, Megson S, Wood D. Iontophoresis as a means of delivering antibiotics into allograft bone (vol 87, pg 1568, 2005). Journal of Bone and Joint Surgery-British Volume.2010; 88B: 837-837.

6. Mankin HJ, Springfield DS, Gebhardt MC, et al. Current status of allografting for bone tumors. Orthopedics.1992; 15: 1147-1154.

7. Bullens PH, Minderhoud NM, de Waal Malefijt MC, et al. Survival of massive allografts in segmental oncological bone defect reconstructions. Int Orthop.2009; 33: 757-760.

8. Lord CF, Gebhardt MC, Tomford WW, et al. Infection in bone allografts. Incidence, nature, and treatment. J Bone Joint Surg Am. 1988; 70: 369-376.

9. Dick HM, Strauch RJ. Infection of massive bone allografts. Clin Orthop Relat Res.1994;306: 46-53.

10. Carano RA, Filvaroff EH. Angiogenesis and bone repair. Drug Discov Today.2003; 8: 980-989

11. Geiger F, Bertram H, Berger I, et al. Vascular endothelial growth factor gene-activated matrix (VEGF165-GAM) enhances osteogenesis and angiogenesis in large segmental bone defects. J Bone Miner Res.2005; 20: 2028-2035.

12. Steffens L, Wenger A, Stark GB, et al. In vivo engineering of a human vasculature for bone tissue engineering applications. J Cell Mol Med.2009;13: 3380-3386.

13. Ketonis C, Barr S, Shapiro IM, et al. Antibacterial activity of bone allografts: comparison of a new vancomycin-tethered allograft with allograft loaded with adsorbed vancomycin. Bone.2011; 48: 631-638.

14. Hou T, Xu J, Li Q, Feng J, Zen L. In vitro evaluation of a fibrin gel antibiotic delivery system containing mesenchymal stem cells and vancomycin alginate beads for treating bone infections and facilitating bone formation. Tissue Eng Part A .2008;14: 1173-1182.

15. Kruyt MC, Dhert WJ, Oner FC, et al. Analysis of ectopic and orthotopic bone formation in cell-based tissue-engineered constructs in goats. Biomaterials.2007; 28: 1798-1805.

16. Wang L, Fan H, Zhang ZY, et al. Osteogenesis and angiogenesis of tissue-engineered bone constructed by prevascularized beta-tricalcium phosphate scaffold and mesenchymal stem cells. Biomaterials.2010;31: 9452-9461.

17. Chan YS, Ueng SW, Wang CJ, et al. Antibiotic-impregnated autogenic cancellous bone grafting is an effective and safe method for the management of small infected tibial defects: a comparison study. J Trauma.2000; 48: 246-255.

18. Kelm J, Bohrer P, Schmitt E,et al. Int J Med Sci. Treatment of proximal femur infections with antibiotic-loaded cement spacers. 2009;6(5):258-64.

19. Ueng SW, Lee SS, Lin SS, et al. Biodegradable alginate antibiotic beads. Clin Orthop Relat Res. 2000;380: 250-259.

20. HOU Tian-yong, Wu Xue-hui, XU Jian-zhong, et al. Preparation and optimization of fibrin-gel-coated vancomycin alginate beads. Chinese Journal of Trauma. 2008; 24: 470-474

21. Fuchs $\mathrm{S}$, Jiang $\mathrm{X}, \mathrm{Schmidt} \mathrm{H}$, et al. Dynamic processes involved in the pre-vascularization of silk fibroin constructs for bone regeneration using outgrowth endothelial cells. Biomaterials.2009; 30: 1329-1338.

22. $\mathrm{Yu} \mathrm{H}$, VandeVord PJ, Mao L, et al. Improved tissue-engineered bone regeneration by endothelial cell mediated vascularization. Biomaterials.2009; 30: 508-517.

23. Boos AM, Loew JS, Deschler G, et al. Directly auto-transplanted mesenchymal stem cells induce bone formation in a ceramic bone substitute in an ectopic sheep model. J Cell Mol Med.2011; 15: 1364-1378.

24. Xing J, Hou T, Luobu B, et al. Anti-Infection Tissue Engineering Construct Treating Osteomyelitis in Rabbit Tibia. Tissue Eng Part A. 2013;19(1-2): 255-263.

25. Edin ML, Miclau T, Lester GE, et al. Effect of cefazolin and vancomycin on osteoblasts in vitro. Clin Orthop Relat Res.1996;333: 245-251.

26. Ramírez E, Jiménez C, Borobia AM, et al. Vancomycin-induced acute kidney injury detected by a prospective pharmacovigilance program from laboratory signals. Ther Drug Monit. 2013; 35(3):360-366. 